\title{
Extension of Lipschitz Functions Defined on Metric Subspaces of Homogeneous Type
}

\author{
Alexander BRUDNYI and Yuri BRUDNYI \\ Department of Mathematics and Statistics \\ Department of Mathematics \\ University of Calgary \\ Technion, Haifa - Israel \\ Calgary - Canada \\ ybrudnyi@math.technion.ac.il \\ albru@math.ucalgary.ca
}

Received: January 1, 2006

Accepted: March 27, 2006

\begin{abstract}
If a metric subspace $M^{o}$ of an arbitrary metric space $M$ carries a doubling measure $\mu$, then there is a simultaneous linear extension of all Lipschitz functions on $M^{o}$ ranged in a Banach space to those on $M$. Moreover, the norm of this linear operator is controlled by logarithm of the doubling constant of $\mu$.
\end{abstract}

Key words: metric space of homogeneous type, Lipschitz function, linear extension.

2000 Mathematics Subject Classification: primary 26B35; secondary 54E35, 46B15.

\section{Formulation of the main result}

Let $(M, d)$ be a metric space and $X$ be a Banach space. The space $\operatorname{Lip}(M, X)$ consists of all $X$-valued Lipschitz functions on $M$. The Lipschitz constant

$$
L(f):=\sup _{m \neq m^{\prime}}\left\{\frac{\left\|f(m)-f\left(m^{\prime}\right)\right\|}{d\left(m, m^{\prime}\right)}: m, m^{\prime} \in M\right\}
$$

of a function $f$ from this space is therefore finite and the function $f \mapsto L(f)$ is a Banach seminorm on $\operatorname{Lip}(M, X)$.

Let $M^{o}$ be a metric subspace of $M$, i.e., $M^{o} \subset M$ is a metric space endowed with the induced metric $\left.d\right|_{M^{\circ} \times M^{\circ}}$.

The first author was supported in part by NSERC. 
Convention. We mark all objects related to the subspace $M^{o}$ by the upper " $O$ ".

A linear operator $E: \operatorname{Lip}\left(M^{o}, X\right) \rightarrow \operatorname{Lip}(M, X)$ is called a simultaneous extension if for all $f \in \operatorname{Lip}\left(M^{o}, X\right)$

$$
\left.E f\right|_{M^{o}}=f
$$

and, moreover, the norm

$$
\|E\|:=\sup \left\{\frac{L(E f)}{L(f)}: f \in \operatorname{Lip}\left(M^{o}, X\right)\right\}
$$

is finite.

To formulate the main result we also need

Definition 1.1. A Borel measure $\mu$ on a metric space $(M, d)$ is said to be doubling if the $\mu$-measure of every open ball

$$
B_{R}(m):=\left\{m^{\prime} \in M: d\left(m, m^{\prime}\right)<R\right\}
$$

is strictly positive and finite and the doubling constant

$$
D(\mu):=\sup \left\{\frac{\mu\left(B_{2 R}(m)\right)}{\mu\left(B_{R}(m)\right)}: m \in M, R>0\right\}
$$

is finite.

A metric space carrying a fixed doubling measure is called of homogeneous type.

Our main result is

Theorem 1.2. Let $M^{o}$ be a metric subspace of an arbitrary metric space $(M, d)$. Assume that $\left(M^{o}, d^{o}\right)$ is of homogeneous type and $\mu^{o}$ is the corresponding doubling measure. Then there exists a simultaneous extension $E: \operatorname{Lip}\left(M^{\circ}, X\right) \rightarrow \operatorname{Lip}(M, X)$ satisfying

$$
\|E\| \leq c\left(\log _{2} D\left(\mu^{o}\right)+1\right)
$$

with some numerical constant $c>1$.

Let us discuss relations of this theorem to some known results. First, a similar result holds for an arbitrary subspace $M^{o}$ provided that the ambient space $M$ is of pointwise homogeneous type, see [1, Theorem 2.21; 2, Theorem 1.14]. The class of metric spaces of pointwise homogeneous type contains, in particular, all metric spaces of homogeneous type, Riemannian manifolds $M_{\omega} \cong \mathbb{R}^{n} \times \mathbb{R}_{+}$with the path metric defined by the Riemannian metric

$$
d s^{2}:=\omega\left(x_{n+1}\right)\left(d x_{1}^{2}+\cdots+d x_{n+1}^{2}\right), \quad\left(x_{1}, \ldots, x_{n}, x_{n+1}\right) \in \mathbb{R}^{n} \times \mathbb{R}_{+},
$$

where $\omega: \mathbb{R}_{+} \rightarrow \mathbb{R}_{+}$is a continuous nonincreasing function (e.g., the hyperbolic spaces $\mathbb{H}^{n}$ are in this class), and finite direct products of these objects.

The following problem is of a considerable interest. 
Problem 1.3. Is it true that Theorem 1.2 is valid for $M^{\circ}(\subset M)$ isometric to a subspace of a metric space $(\hat{M}, \hat{d})$ of pointwise homogeneous type with $\|E\| \leq c(\hat{M})$ ? (Here $c(\hat{M})$ depends on some characteristics of $\hat{M}$ only.)

It is proved in [2] that as such $M^{o}$ one can take, e.g., finite direct products of Gromov hyperbolic spaces of bounded geometry and that the answer in Problem 1.3 is positive in this case.

Second, as a consequence of Theorem 1.2 we obtain a deep extension result due to Lee and Naor, see [4, Theorem 1.6]. The latter asserts that a simultaneous extension $E: \operatorname{Lip}\left(M^{o}, X\right) \rightarrow \operatorname{Lip}(M, X)$ exists whenever the subspace $\left(M^{o}, d^{o}\right)$ of $(M, d)$ has the finite doubling constant $\delta\left(M^{o}\right)$ and, moreover,

$$
\|E\| \leq c \log _{2} \delta\left(M^{o}\right)
$$

with some numerical constant $c>1$.

Let us recall that the doubling constant $\delta(M)$ of a metric space $(M, d)$ is the infimum of integers $N$ such that every closed ball of $M$ of radius $R$ can be covered by $N$ closed balls of radius $R / 2$. The space $M$ is said to be doubling if $\delta(M)<\infty$.

To derive the Lee-Naor theorem from our main result we first note that without loss of generality one may assume that $\left(M^{o}, d^{o}\right)$ is complete. By the Koniagin-Vol'berg theorem [6] (see also [5]) a complete doubling space $M$ carries a doubling measure $\mu$ such that

$$
\log _{2} D(\mu) \leq c \log _{2} \delta(M)
$$

where $c \geq 1$ is a numerical constant. Together with (2) this implies the Naor-Lee result.

On the other hand, it was noted in [3] that if $M$ carries a doubling measure $\mu$, then this space is doubling and

$$
\log _{2} \delta(M) \leq c \log _{2} D(\mu)
$$

with some numerical constant $c>1$. Hence, Theorem 1.2 is, in turn, a consequence of (3) and the Lee-Naor theorem. However, the rather elaborated proof of the latter result is nonconstructive. (It exploits an appropriate stochastic metric decomposition of $M \backslash M^{\circ}$.) In contrast, our proof is constructive and is based on a simple average procedure. Therefore our proof can be also seen as a streamlining constructive method of the proof of the Lee-Naor theorem.

\section{Proof of Theorem 1.2}

We begin with the following remark reducing the required result to a special case.

Let $M$ and $M^{o}$ be isometric to subspaces of a new metric space $\hat{M}$ and its subspace $\hat{M}^{o}$, respectively. Assume that there exists a simultaneous extension $\hat{E}$ : 
$\operatorname{Lip}\left(\hat{M}^{o}, X\right) \rightarrow \operatorname{Lip}(\hat{M}, X)$. Then, after identification of $M^{o}$ and $M$ with the corresponding isometric subspaces of $\hat{M}$, the operator $\hat{E}$ gives rise to a simultaneous extension $E: \operatorname{Lip}\left(M^{o}, X\right) \rightarrow \operatorname{Lip}(M, X)$ satisfying

$$
\|E\| \leq\|\hat{E}\| \text {. }
$$

If, in addition, $\|\hat{E}\|$ is bounded by the right-hand side of (2), then the desired result immediately follows.

We choose as the above pair $\hat{M}^{o} \subset \hat{M}$ metric spaces denoted by $M_{N}^{o}$ and $M_{N}$ where $N \geq 1$ is a fixed integer and defined as follows.

The underlying sets of these spaces are

$$
M_{N}:=M \times \mathbb{R}^{N}, \quad M_{N}^{o}:=M^{o} \times \mathbb{R}^{N} ;
$$

a metric $d_{N}$ on $M_{N}$ is given by

$$
d_{N}\left((m, x),\left(m^{\prime}, x^{\prime}\right)\right):=d\left(m, m^{\prime}\right)+\left|x-x^{\prime}\right|_{1}
$$

where $m, m^{\prime} \in M$ and $x, x^{\prime} \in \mathbb{R}^{N}$, and $|x|_{1}:=\sum_{i=1}^{N}\left|x_{i}\right|$ is the $l_{1}^{N}$-metric of $x \in \mathbb{R}^{N}$. Further, $d_{N}^{o}$ denotes the metric on $M_{N}^{o}$ induced by $d_{N}$.

Finally, we define a Borel measure $\mu_{N}^{o}$ on $M_{N}^{o}$ as the tensor product of the measure $\mu^{o}$ and the Lebesgue measure $\lambda_{N}$ on $\mathbb{R}^{N}$ :

$$
\mu_{N}^{o}:=\mu^{o} \otimes \lambda_{N}
$$

We extend this measure to the $\sigma$-algebra consisting of subsets $S \subset M_{N}$ such that $S \cap M_{N}^{o}$ is a Borel subset of $M_{N}^{o}$. Namely, we set for these $S$

$$
\bar{\mu}_{N}(S):=\mu_{N}^{o}\left(S \cap M_{N}^{o}\right) .
$$

It is important for the subsequent part of the proof that every open ball $B_{R}((m, x)) \subset$ $M_{N}$ belongs to this $\sigma$-algebra. In fact, its intersection with $M_{N}^{o}$ is a Borel subset of this space, since the function $\left(m^{\prime}, x^{\prime}\right) \mapsto d_{N}\left((m, x),\left(m^{\prime}, x^{\prime}\right)\right)$ is continuous on $M_{N}^{o}$. Hence,

$$
\bar{\mu}_{N}\left(B_{R}((m, x))\right)=\mu_{N}^{o}\left(B_{R}((m, x)) \cap M_{N}^{o}\right) .
$$

Auxiliary results. The measure $\mu_{N}^{o}$ is clearly doubling. Therefore its dilation function given for $l \geq 1$ by

$$
D_{N}^{o}(l):=\sup \left\{\frac{\mu_{N}^{o}\left(B_{l R}^{o}(\hat{m})\right)}{\mu_{N}^{o}\left(B_{R}^{o}(\hat{m})\right)}: \hat{m} \in M_{N}^{o} \text { and } R>0\right\}
$$

is finite.

Hereafter we denote by $\hat{m}$ the pair $(m, x)$ with $m \in M$ and $x \in \mathbb{R}^{N}$, and by $B_{R}^{o}(\hat{m})$ the open ball in $M_{N}^{o}$ centered at $\hat{m} \in M_{N}^{o}$ and of radius $R$. The open ball $B_{R}(\hat{m})$ of $M_{N}$ relates to that by

$$
B_{R}^{o}(\hat{m})=B_{R}(\hat{m}) \cap M_{N}^{o}
$$


provided $\hat{m} \in M_{N}^{o}$.

In [1] the value $D_{N}^{o}(1+1 / N)$ is proved to be bounded by some numerical constant for all sufficiently large $N$. In the argument presented below we require a similar estimate for a (modified) dilation function $D_{N}$ for the extended measure $\bar{\mu}_{N}$. This is given for $l \geq 1$ by

$$
D_{N}(l):=\sup \left\{\frac{\bar{\mu}_{N}\left(B_{l R}(\hat{m})\right)}{\bar{\mu}_{N}\left(B_{R}(\hat{m})\right)}\right\}
$$

where the supremum is taken over all $R$ satisfying

$$
R>4 d\left(\hat{m}, M_{N}^{o}\right):=4 \inf \left\{d_{N}\left(\hat{m}, \hat{m}^{\prime}\right): \hat{m}^{\prime} \in M_{N}^{o}\right\}
$$

and then over all $\hat{m} \in M_{N}$.

Due to (4) and (6) the denominator in (5) is not zero and $D_{N}(l)$ is well defined.

Comparison of the above dilation functions shows that $D_{N}^{o}(l) \leq D_{N}(l)$. Nevertheless, the converse is also true for $l$ close to 1 .

Lemma 2.1. Assume that $N$ and the doubling constant $D:=D\left(\mu^{o}\right)$, see (1), are related by

$$
N \geq\left[3 \log _{2} D\right]+5 .
$$

Then the following is true:

$$
D_{N}(1+1 / N) \leq \frac{6}{5} e^{4} .
$$

Proof. In accordance with the definition of $D_{N}$, see (5), we must estimate the function

$$
\frac{\bar{\mu}_{N}\left(B_{R_{N}}(\hat{m})\right)}{\bar{\mu}_{N}\left(B_{R}(\hat{m})\right)} \quad \text { where } \quad R_{N}:=\left(1+\frac{1}{N}\right) R .
$$

Since the points $\hat{m}^{\prime}$ of the ball $B_{R_{N}}(\hat{m})$ of $M_{N}$ satisfy the inequality

$$
d\left(m, m^{\prime}\right)+\left|x-x^{\prime}\right|_{1}<R_{N},
$$

the Fubini theorem and (4) yield

$$
\bar{\mu}_{N}\left(B_{R_{N}}(\hat{m})\right)=\gamma_{N} \int_{M^{o} \cap B_{R_{N}}(m)}\left(R_{N}-d\left(m, m^{\prime}\right)\right)^{N} d \mu^{o}\left(m^{\prime}\right) .
$$

Here $\gamma_{N}$ is the volume of the unit $l_{1}^{N}$-ball.

We must estimate the integral in (9) from above under the condition

$$
d_{N}\left(\hat{m}, M_{N}^{o}\right)<R / 4 .
$$

To this end split the integral into one over $B_{3 R / 4}(m) \cap M^{o}$ and one over the remaining part $\left(B_{R_{N}}(m) \backslash B_{3 R / 4}(m)\right) \cap M^{o}$. Denote these integrals by $I_{1}$ and $I_{2}$. For $I_{2}$ we get

$$
I_{2} \leq \gamma_{N}\left(R_{N}-3 R / 4\right)^{N} \mu^{o}\left(B_{R_{N}}(m) \cap M^{o}\right) .
$$


Further, from (10) we clearly have

$$
d\left(m, M^{o}\right)<R / 4 .
$$

Pick a point $\tilde{m} \in M^{o}$ so that

$$
d\left(m, M^{o}\right) \leq d(m, \tilde{m})<R / 4 .
$$

Then we have the following embeddings

$$
B_{R_{N} / 4}^{o}(\tilde{m}) \subset B_{R_{N} / 2}(m) \cap M^{o} \subset B_{R_{N}}(m) \cap M^{o} \subset B_{5 R_{N} / 4}^{o}(\tilde{m}) .
$$

Applying the doubling inequality for the measure $\mu^{o}$, see (1), we then obtain

$$
\mu^{o}\left(B_{R_{N}}(m) \cap M^{o}\right) \leq D^{3} \mu^{o}\left(B_{R_{N} / 2}(m) \cap M^{o}\right) .
$$

Moreover, due to (7)

$$
D^{3}<2^{\left[3 \log _{2} D\right]+1} \leq 2^{N-4} .
$$

Combining the last two inequalities with (11) we have

$$
I_{2} \leq \gamma_{N} 2^{-N-4}\left(1+\frac{4}{N}\right)^{N} R^{N} \mu^{o}\left(B_{R_{N} / 2}(m) \cap M^{o}\right) .
$$

To estimate the integral $I_{1}$ we rewrite its integrand as follows:

$$
\left(R_{N}-d\left(m, m^{\prime}\right)\right)^{N}=\left(1+\frac{1}{N}\right)^{N}\left(R-d\left(m, m^{\prime}\right)\right)^{N}\left(1+\frac{d\left(m, m^{\prime}\right)}{(N+1)\left(R-d\left(m, m^{\prime}\right)\right)}\right)^{N} .
$$

Since $m^{\prime} \in B_{3 / 4 R}(m)$, the last factor is at most $\left(1+\frac{3 R / 4}{(N+1) R / 4}\right)^{N}=\left(1+\frac{3}{N+1}\right)^{N}$. This yields

$$
\begin{aligned}
I_{1} & \leq \gamma_{N}\left(1+\frac{1}{N}\right)^{N}\left(1+\frac{3}{N+1}\right)^{N} \int_{B_{3 R / 4}(m) \cap M^{o}}\left(R-d\left(m, m^{\prime}\right)\right)^{N} d \mu^{o}\left(m^{\prime}\right) \\
& \leq e^{4} \bar{\mu}_{N}\left(B_{R}(\hat{m})\right) .
\end{aligned}
$$

Hence for the part of fraction (8) related to $I_{1}$ we have

$$
\tilde{I}_{1}:=\frac{I_{1}}{\bar{\mu}_{N}\left(B_{R}(\hat{m})\right)} \leq e^{4} .
$$

To estimate the remaining part $\tilde{I}_{2}:=\frac{I_{2}}{\bar{\mu}_{N}\left(B_{R}(\hat{m})\right)}$ we note that its denominator is greater than

$$
\gamma_{N} \int_{M^{o} \cap B_{R_{N} / 2}(m)}\left(R-d\left(m, m^{\prime}\right)\right)^{N} d \mu^{o}\left(m^{\prime}\right) .
$$


Since here $d\left(m, m^{\prime}\right) \leq R_{N} / 2$, this, in turn, is bounded from below by

$$
\gamma_{N} 2^{-N}\left(1-\frac{1}{N}\right)^{N} R^{N} \mu^{o}\left(B_{R_{N} / 2}(m) \cap M^{o}\right) .
$$

Combining this with (12) and noting that $N \geq 5$ we get

$$
\tilde{I}_{2} \leq 2^{-4}\left(1-\frac{1}{N}\right)^{-N}\left(1+\frac{4}{N}\right)^{N}<\frac{1}{5} e^{4} .
$$

Hence the fraction (8) is bounded by $\tilde{I}_{1}+\tilde{I}_{2} \leq \frac{6}{5} e^{4}$, see (13), and this immediately implies the required estimate of $D_{N}(1+1 / N)$.

In the next lemma we estimate $\bar{\mu}_{N}$-measure of the spherical layer $B_{R_{2}}(\hat{m})-B_{R_{1}}(\hat{m})$, $R_{2} \geq R_{1}$, by a kind of a surface measure. For its formulation we set

$$
A_{N}:=\frac{12}{5} e^{4} N
$$

Lemma 2.2. Assume that

$$
N \geq\left[3 \log _{2} D\right]+6 .
$$

Then for all $\hat{m} \in M_{N}$ and $R_{1}, R_{2}>0$ satisfying

$$
R_{2} \geq \max \left\{R_{1}, 8 d_{N}\left(\hat{m}, M_{N}^{o}\right)\right\}
$$

the following is true

$$
\bar{\mu}_{N}\left(B_{R_{2}}(\hat{m}) \backslash B_{R_{1}}(\hat{m})\right) \leq A_{N} \frac{\bar{\mu}_{N}\left(B_{R_{2}}(\hat{m})\right)}{R_{2}}\left(R_{2}-R_{1}\right) .
$$

Proof. By definition $M_{N}=M_{N-1} \times \mathbb{R}$ and $\bar{\mu}_{N}=\bar{\mu}_{N-1} \otimes \lambda_{1}$. Then by the Fubini theorem we have for $R_{1} \leq R_{2}$ with $\hat{m}=(\tilde{m}, t)$

$$
\begin{aligned}
\bar{\mu}_{N}\left(B_{R_{2}}(\hat{m})\right)-\bar{\mu}_{N}\left(B_{R_{1}}(\hat{m})\right) & =2 \int_{R_{1}}^{R_{2}} \bar{\mu}_{N-1}\left(B_{s}(\tilde{m})\right) d s \\
& \leq \frac{2 R_{2} \bar{\mu}_{N-1}\left(B_{R_{2}}(\tilde{m})\right)}{R_{2}}\left(R_{2}-R_{1}\right) .
\end{aligned}
$$

We claim that for arbitrary $l>1$ and $R \geq 8 d_{N}\left(\hat{m}, M_{N}^{o}\right):=8 d_{N-1}\left(\tilde{m}, M_{N-1}^{o}\right)$

$$
R \bar{\mu}_{N-1}\left(B_{R}(\tilde{m})\right) \leq \frac{l D_{N-1}(l)}{l-1} \bar{\mu}_{N}\left(B_{R}(\hat{m})\right) .
$$

Together with the previous inequality this will yield

$$
\bar{\mu}_{N}\left(B_{R_{2}}(\hat{m})\right)-\bar{\mu}_{N}\left(B_{R_{1}}(\hat{m})\right) \leq \frac{2 l D_{N-1}(l)}{l-1} \cdot \frac{\bar{\mu}_{N}\left(B_{R_{2}}(\hat{m})\right)}{R_{2}}\left(R_{2}-R_{1}\right) .
$$


Finally choose here $l=1+\frac{1}{N-1}$ and use Lemma 2.1. This will give the required inequality.

Hence, it remains to establish (15). By the definition of $D_{N-1}(l)$ we have for $l>1$ using the previous lemma

$$
\begin{aligned}
\bar{\mu}_{N}\left(B_{l R}(\hat{m})\right) & =2 l \int_{0}^{R} \bar{\mu}_{N-1}\left(B_{l s}(\tilde{m})\right) d s \leq 4 l \int_{R / 2}^{R} \bar{\mu}_{N-1}\left(B_{l s}(\tilde{m})\right) d s \\
& \leq 4 l D_{N-1}(l) \int_{R / 2}^{R} \bar{\mu}_{N-1}\left(B_{s}(\tilde{m})\right) d s \leq 2 l D_{N-1}(l) \bar{\mu}_{N}\left(B_{R}(\hat{m})\right) .
\end{aligned}
$$

On the other hand, replacing $[0, R]$ by $\left[l^{-1} R, R\right]$ we also have

$$
\bar{\mu}_{N}\left(B_{l R}(\hat{m})\right) \geq 2 l \bar{\mu}_{N-1}\left(B_{R}(\tilde{m})\right)\left(R-l^{-1} R\right)=2(l-1) R \bar{\mu}_{N-1}\left(B_{R}(\tilde{m})\right) .
$$

Combining the last two inequalities we get (15).

Extension operator. We define the required simultaneous extension

$$
E: \operatorname{Lip}\left(M_{N}^{o}, X\right) \rightarrow \operatorname{Lip}\left(M_{N}, X\right)
$$

using the standard average operator Ave defined on continuous and locally bounded functions $g: M_{N}^{o} \rightarrow X$ by

$$
\operatorname{Ave}(g ; \hat{m}, R):=\frac{1}{\bar{\mu}_{N}\left(B_{R}(\hat{m})\right)} \int_{B_{R}(\hat{m})} g d \bar{\mu}_{N} .
$$

To be well-defined the domain of integration $B_{R}(\hat{m}) \cap M_{N}^{o}$ should be of strictly positive $\bar{\mu}_{N}$-measure (i.e., $\mu_{N}^{o}$-measure). This condition is fulfilled in the case presented now. Namely, we define the simultaneous extension $E$ on functions $f \in \operatorname{Lip}\left(M_{N}^{o}, X\right)$ by

$$
(E f)(\hat{m}):=\left\{\begin{array}{lll}
f(\hat{m}) & \text { if } & \hat{m} \in M_{N}^{o}, \\
\operatorname{Ave}(f ; m, R(\hat{m})) & \text { if } & \hat{m} \notin M_{N}^{o}
\end{array}\right.
$$

where we set

$$
R(\hat{m}):=8 d_{N}\left(\hat{m}, M_{N}^{o}\right) .
$$

The required estimate of $\|E\|$ is presented below. To formulate the result we set

$$
K_{N}(l):=A_{N} D_{N}(l)(4 l+1)
$$

where the first of two factors are defined by (14) and (5).

Proposition 2.3. The following inequality,

$$
\|E\| \leq 20 A_{N}+\max \left(\frac{4 l+1}{2(l-1)}, K_{N}(l)\right),
$$

is true provided $l:=1+1 / N$. 
Before we begin the proof let us derive from here the desired result. Namely, choose

$$
N:=\left[3 \log _{2} D\right]+6
$$

and use Lemma 2.1 and (14) to estimate $D_{N}(1+1 / N)$ and $A_{N}$. Then we get

$$
\|E\| \leq C\left(\log _{2} D+2\right)
$$

with some numerical constant $C$. This clearly gives (2).

Proof. We have to show that for every $\hat{m}_{1}, \hat{m}_{2} \in M_{N}$

$$
\left\|(E f)\left(\hat{m}_{1}\right)-(E f)\left(\hat{m}_{2}\right)\right\|_{X} \leq K\|f\|_{\operatorname{Lip}\left(M_{N}, X\right)} d_{N}\left(\hat{m}_{1}, \hat{m}_{2}\right)
$$

where $K$ is the constant in the inequality of the proposition.

It suffices to consider only two cases:

(i) $\hat{m}_{1} \in M_{N}^{o}$ and $\hat{m}_{2} \notin M_{N}^{o}$,

(ii) $\hat{m}_{1}, \hat{m}_{2} \notin M_{N}^{o}$.

We assume without loss of generality that

$$
\|f\|_{\operatorname{Lip}\left(M_{N}^{o}, X\right)}=1
$$

and simplify the computations by introducing the following notations:

$$
R_{i}:=d_{N}\left(\hat{m}_{i}, M_{N}^{o}\right), \quad B_{i j}:=B_{8 R_{j}}\left(\hat{m}_{i}\right), \quad v_{i j}:=\bar{\mu}_{N}\left(B_{i j}\right), \quad 1 \leq i, j \leq 2 .
$$

We assume also for definiteness that

$$
0<R_{1} \leq R_{2} .
$$

By the triangle inequality we then have

$$
0 \leq R_{2}-R_{1} \leq d_{N}\left(\hat{m}_{1}, \hat{m}_{2}\right) .
$$

Further, by Lemma 2.2 the quantities introduced satisfy the following inequality:

$$
v_{i 2}-v_{i 1} \leq \frac{A_{N} v_{i 2}}{R_{2}}\left(R_{2}-R_{1}\right) .
$$

Let now $\hat{m}^{*}$ be such that $d_{N}\left(\hat{m}_{1}, \hat{m}^{*}\right)<2 R_{1}$. Set

$$
\hat{f}(\hat{m}):=f(\hat{m})-f\left(\hat{m}^{*}\right) .
$$

From the triangle inequality we then obtain

$$
\max \left\{\|\hat{f}(\hat{m})\|_{X}: \hat{m} \in B_{i 2} \cap M_{N}^{o}\right\} \leq 10 R_{2}+(i-1) d_{N}\left(\hat{m}_{1}, \hat{m}_{2}\right) .
$$


(Here $i=1,2$.)

We now prove (18) for $\hat{m}_{1} \in M_{N}^{o}$ and $\hat{m}_{2} \notin M_{N}^{o}$. We begin with the evident inequality

$$
\left\|(E f)\left(\hat{m}_{2}\right)-(E f)\left(\hat{m}_{1}\right)\right\|_{X}=\frac{1}{v_{22}}\left\|\int_{B_{22}} \hat{f}(\hat{m}) d \bar{\mu}_{N}\right\|_{X} \leq \max _{B_{22} \cap M_{N}^{o}}\|\hat{f}\|_{X},
$$

see (19) and (22). Applying (23) with $i=2$ we then bound this maximum by $10 R_{2}+d_{N}\left(\hat{m}_{1}, \hat{m}_{2}\right)$. But $\hat{m}_{1} \in M_{N}^{o}$ and so

$$
R_{2}=d_{N}\left(\hat{m}_{2}, M_{N}^{o}\right) \leq d_{N}\left(\hat{m}_{1}, \hat{m}_{2}\right) ;
$$

therefore (18) holds in this case with $K=11$.

The remaining case $\hat{m}_{1}, \hat{m}_{2} \notin M_{N}^{o}$ requires some additional auxiliary results. For their formulations we first write

$$
(E f)\left(\hat{m}_{1}\right)-(E f)\left(\hat{m}_{2}\right):=D_{1}+D_{2}
$$

where

$$
\begin{aligned}
& D_{1}:=\operatorname{Ave}\left(\hat{f} ; \hat{m}_{1}, 8 R_{1}\right)-\operatorname{Ave}\left(\hat{f} ; \hat{m}_{1}, 8 R_{2}\right), \\
& D_{2}:=\operatorname{Ave}\left(\hat{f} ; \hat{m}_{1}, 8 R_{2}\right)-\operatorname{Ave}\left(\hat{f} ; \hat{m}_{2}, 8 R_{2}\right),
\end{aligned}
$$

see (16) and (22).

Lemma 2.4. We have

$$
\left\|D_{1}\right\|_{X} \leq 20 A_{N} d_{N}\left(\hat{m}_{1}, \hat{m}_{2}\right) .
$$

Recall that $A_{N}$ is the constant defined by (14).

Proof. By (25), (22), and (19),

$$
\begin{aligned}
D_{1} & =\frac{1}{v_{11}} \int_{B_{11}} \hat{f} d \bar{\mu}_{N}-\frac{1}{v_{12}} \int_{B_{12}} \hat{f} d \bar{\mu}_{N} \\
& =\left(\frac{1}{v_{11}}-\frac{1}{v_{12}}\right) \int_{B_{11}} \hat{f} d \bar{\mu}_{N}-\frac{1}{v_{12}} \int_{B_{12} \backslash B_{11}} \hat{f} d \bar{\mu}_{N} .
\end{aligned}
$$

This immediately implies that

$$
\left\|D_{1}\right\|_{X} \leq 2 \cdot \frac{v_{12}-v_{11}}{v_{12}} \cdot \max _{B_{12} \cap M_{N}^{o}}\|\hat{f}\|_{X} .
$$

Applying now (21) and (20), and then (23) with $i=1$ we get the desired estimate.

To obtain a similar estimate for $D_{2}$ we will use the following two facts. 
Lemma 2.5. Assume that for a given $l>1$

$$
d_{N}\left(\hat{m}_{1}, \hat{m}_{2}\right) \leq 8(l-1) R_{2} .
$$

Let for definiteness

$$
v_{22} \leq v_{12}
$$

Then we have

$$
\bar{\mu}_{N}\left(B_{12} \Delta B_{22}\right) \leq A_{N} D_{N}(l) \frac{v_{12}}{4 R_{2}} d_{N}\left(\hat{m}_{1}, \hat{m}_{2}\right) .
$$

(Here $\Delta$ denotes symmetric difference of sets.)

Proof. Set

$$
R:=8 R_{2}+d_{N}\left(\hat{m}_{1}, \hat{m}_{2}\right) .
$$

Then $B_{12} \cup B_{22} \subset B_{R}\left(\hat{m}_{1}\right) \cap B_{R}\left(\hat{m}_{2}\right)$, and

$$
\begin{aligned}
\bar{\mu}_{N}\left(B_{12} \Delta B_{22}\right) \leq\left(\bar{\mu}_{N}\left(B_{R}\left(\hat{m}_{1}\right)\right)-\bar{\mu}_{N}\left(B_{8 R_{2}}\left(\hat{m}_{1}\right)\right)\right) & \\
& +\left(\bar{\mu}_{N}\left(B_{R}\left(\hat{m}_{2}\right)\right)-\bar{\mu}_{N}\left(B_{8 R_{2}}\left(\hat{m}_{2}\right)\right)\right) .
\end{aligned}
$$

Estimating the terms on the right-hand side by Lemma 2.2 we bound them by

$$
A_{N} \frac{\bar{\mu}_{N}\left(B_{R}\left(\hat{m}_{1}\right)\right)}{R}\left(R-8 R_{2}\right)+A_{N} \frac{\bar{\mu}_{N}\left(B_{R}\left(\hat{m}_{2}\right)\right)}{R}\left(R-8 R_{2}\right) .
$$

Moreover, $8 R_{2} \leq R \leq 8 l R_{2}$ and $R-8 R_{2}:=d_{N}\left(\hat{m}_{1}, \hat{m}_{2}\right)$, see (26); taking into account (5), (19), and (27) we therefore have

$$
\bar{\mu}_{N}\left(B_{12} \Delta B_{22}\right) \leq A_{N} D_{N}(l) \frac{v_{12}}{4 R_{2}} d_{N}\left(\hat{m}_{1}, \hat{m}_{2}\right) .
$$

Lemma 2.6. Under the assumptions of the previous lemma we have

$$
v_{12}-v_{22} \leq A_{N} D_{N}(l) \frac{v_{12}}{4 R_{2}} d_{N}\left(\hat{m}_{1}, \hat{m}_{2}\right)
$$

Proof. By (19) the left-hand side is bounded by $\bar{\mu}_{N}\left(B_{12} \Delta B_{22}\right)$.

We now estimate $D_{2}$ from (25) beginning with

Lemma 2.7. Under the conditions of Lemma 2.5 we have

$$
\left\|D_{2}\right\|_{X} \leq K_{N}(l) d_{N}\left(\hat{m}_{1}, \hat{m}_{2}\right)
$$

where $K_{N}(l):=A_{N} D_{N}(l)(4 l+1)$. 
Proof. By the definition of $D_{2}$ and our notation, see (25), (22), and (19),

$$
\begin{aligned}
\left\|D_{2}\right\|_{X} & :=\left\|\frac{1}{v_{12}} \int_{B_{12}} \hat{f} d \bar{\mu}_{N}-\frac{1}{v_{22}} \int_{B_{22}} \hat{f} d \bar{\mu}_{N}\right\|_{X} \\
& \leq \frac{1}{v_{12}} \int_{B_{12} \Delta B_{22}}\|\hat{f}\|_{X} d \bar{\mu}_{N}+\left|\frac{1}{v_{12}}-\frac{1}{v_{22}}\right| \int_{B_{22}}\|\hat{f}\|_{X} d \bar{\mu}_{N}:=J_{1}+J_{2} .
\end{aligned}
$$

By (28), (26), and (23)

$$
\begin{aligned}
J_{1} & \leq \frac{1}{v_{12}} \bar{\mu}_{N}\left(B_{12} \Delta B_{22}\right) \sup _{\left(B_{12} \Delta B_{22}\right) \cap M_{N}^{o}}\|\hat{f}\|_{X} \\
& \leq \frac{A_{N} D_{N}(l)}{4 R_{2}} d_{N}\left(\hat{m}_{1}, \hat{m}_{2}\right)\left(d_{N}\left(\hat{m}_{1}, \hat{m}_{2}\right)+10 R_{2}\right) \\
& \leq A_{N} D_{N}(l)(2 l+1 / 2) d_{N}\left(\hat{m}_{1}, \hat{m}_{2}\right) .
\end{aligned}
$$

Also, (29), (23), and (26) yield

$$
J_{2} \leq A_{N} D_{N}(l)(2 l+1 / 2) d_{N}\left(\hat{m}_{1}, \hat{m}_{2}\right) .
$$

Combining these we get the required estimate.

It remains to consider the case of $\hat{m}_{1}, \hat{m}_{2} \notin M_{N}$ satisfying the inequality

$$
d_{N}\left(\hat{m}_{1}, \hat{m}_{2}\right)>8(l-1) R_{2}
$$

converse to (26). Now the definition (25) of $D_{2}$ and (23) imply that

$$
\left\|D_{2}\right\|_{X} \leq 2 \sup _{\left(B_{12} \cup B_{22}\right) \cap M_{N}^{o}}\|\hat{f}\|_{X} \leq 2\left(10 R_{2}+d_{N}\left(\hat{m}_{1}, \hat{m}_{2}\right)\right) \leq \frac{4 l+1}{2(l-1)} d_{N}\left(\hat{m}_{1}, \hat{m}_{2}\right) .
$$

Combining this with the inequalities of Lemmas 2.4 and 2.7 and equality (24) we obtain the required estimate of the Lipschitz norm of the extension operator $E$ :

$$
\|E\| \leq 20 A_{N}+\max \left(\frac{4 l+1}{2(l-1)}, K_{N}(l)\right)
$$

where $K_{N}(l)$ is the constant in (17).

\section{References}

[1] A. Brudnyi and Yu. Brudnyi, Metric spaces with linear extensions preserving Lipschitz condition, Amer. J. Math, to appear.

[2] _ A universal Lipschitz extension property of Gromov hyperbolic spaces, Rev. Mat. Iberoamericana, to appear. 
[3] R. R. Coifman and G. Weiss, Extensions of Hardy spaces and their use in analysis, Bull. Amer. Math. Soc. 83 (1977), no. 4, 569-645.

[4] J. R. Lee and A. Naor, Extending Lipschitz functions via random metric partitions, Invent. Math. 160 (2005), no. 1, 59-95.

[5] J. Luukkainen and E. Saksman, Every complete doubling metric space carries a doubling measure, Proc. Amer. Math. Soc. 126 (1998), no. 2, 531-534.

[6] A. L. Vol'berg and S. V. Konyagin, On measures with the doubling condition, Izv. Akad. Nauk SSSR Ser. Mat. 51 (1987), no. 3, 666-675 (Russian); English transl., Math. USSR-Izv. 30 (1988), no. $3,629-638$. 